\title{
Fully-Printed, All-Polymer, Bendable and Highly Transparent Complementary Logic Circuits
}

Saumen Mandal a , Giorgio Dell'Erba ${ }^{\mathrm{a}, \mathrm{c}}$, Alessandro Luzio a, Sadir Gabriele Bucella ${ }^{\mathrm{a}, \mathrm{b}}$, Andrea Perinot $^{\mathrm{a}, \mathrm{b}}$, Alberto Calloni ${ }^{\mathrm{b}}$, Giulia Berti ${ }^{\mathrm{b}}$, Gianlorenzo Bussetti ${ }^{\mathrm{b}}$, Lamberto Duò ${ }^{\mathrm{b}}$, Antonio Facchetti $^{\mathrm{d}}$, Yong-Young Noh ${ }^{\mathrm{e}}$, and Mario Caironi ${ }^{\mathrm{a}, *}$

${ }^{a}$ Center for Nano Science and Technology @PoliMi, Istituto Italiano di Tecnologia, Via Pascoli 70/3,20133, Milano, Italy

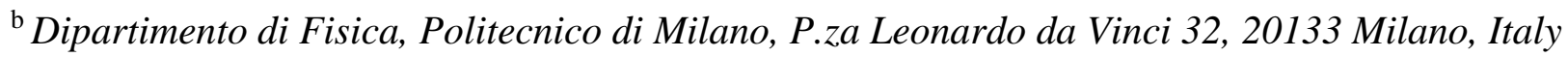

${ }^{\mathrm{c}}$ Dipartimento di Elettronica e Informazione, Politecnico di Milano, P.za Leonardo da Vinci 32, 20133 Milano, Italy

${ }^{\mathrm{d}}$ Polyera Corporation, 8045 Lamon Avenue, Skokie, Illinois 60077, USA

e Department of Energy and Materials Engineering, Dongguk University, 26 Pil-dong, 3 ga, Jung-gu, Seoul 100-715, Republic of Korea 


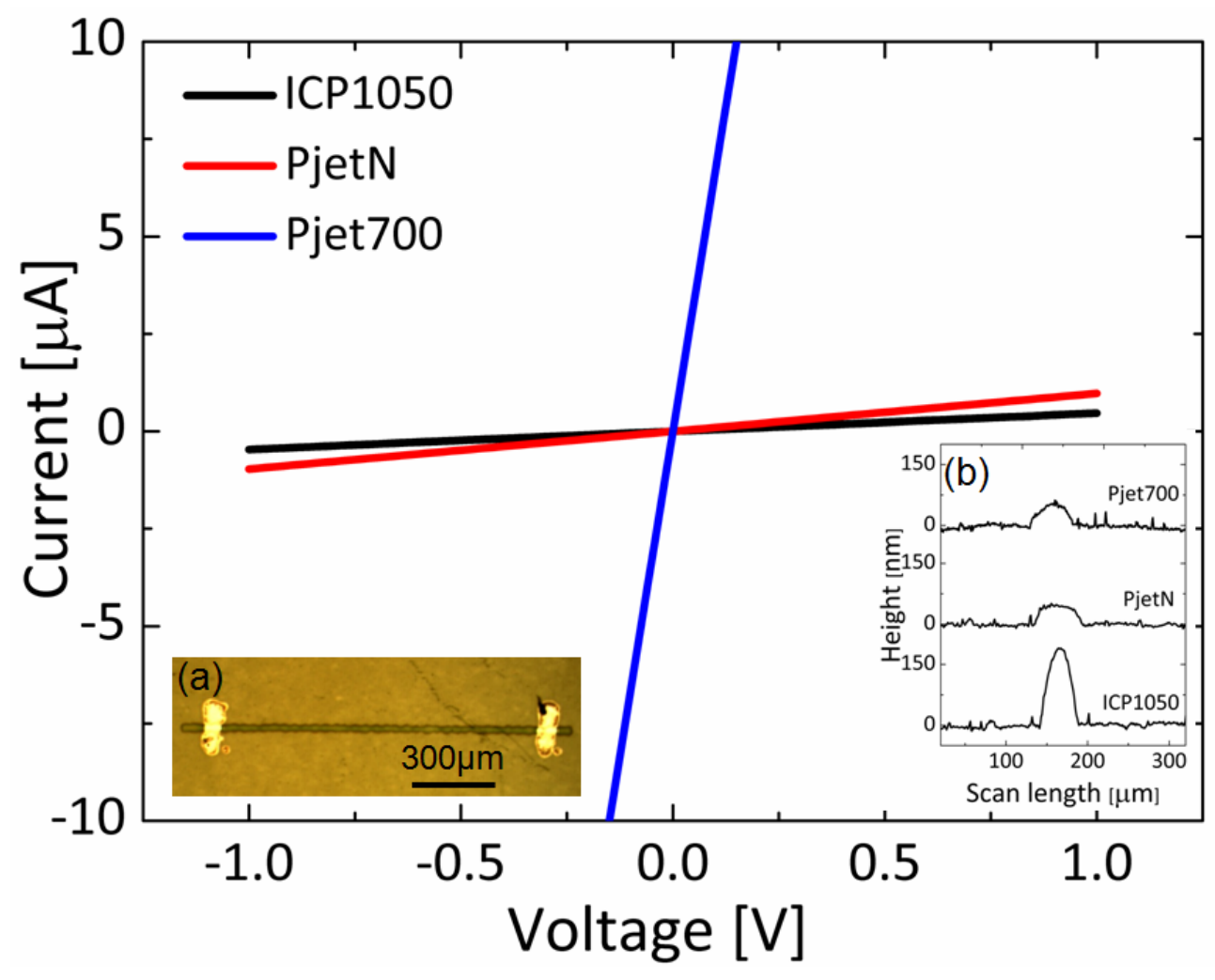

Figure S1. Current-voltage characteristics of all three different PEDOT:PSS printed lines. Inset (a) optical micrograph and (b) thickness profiles of the printed lines. 

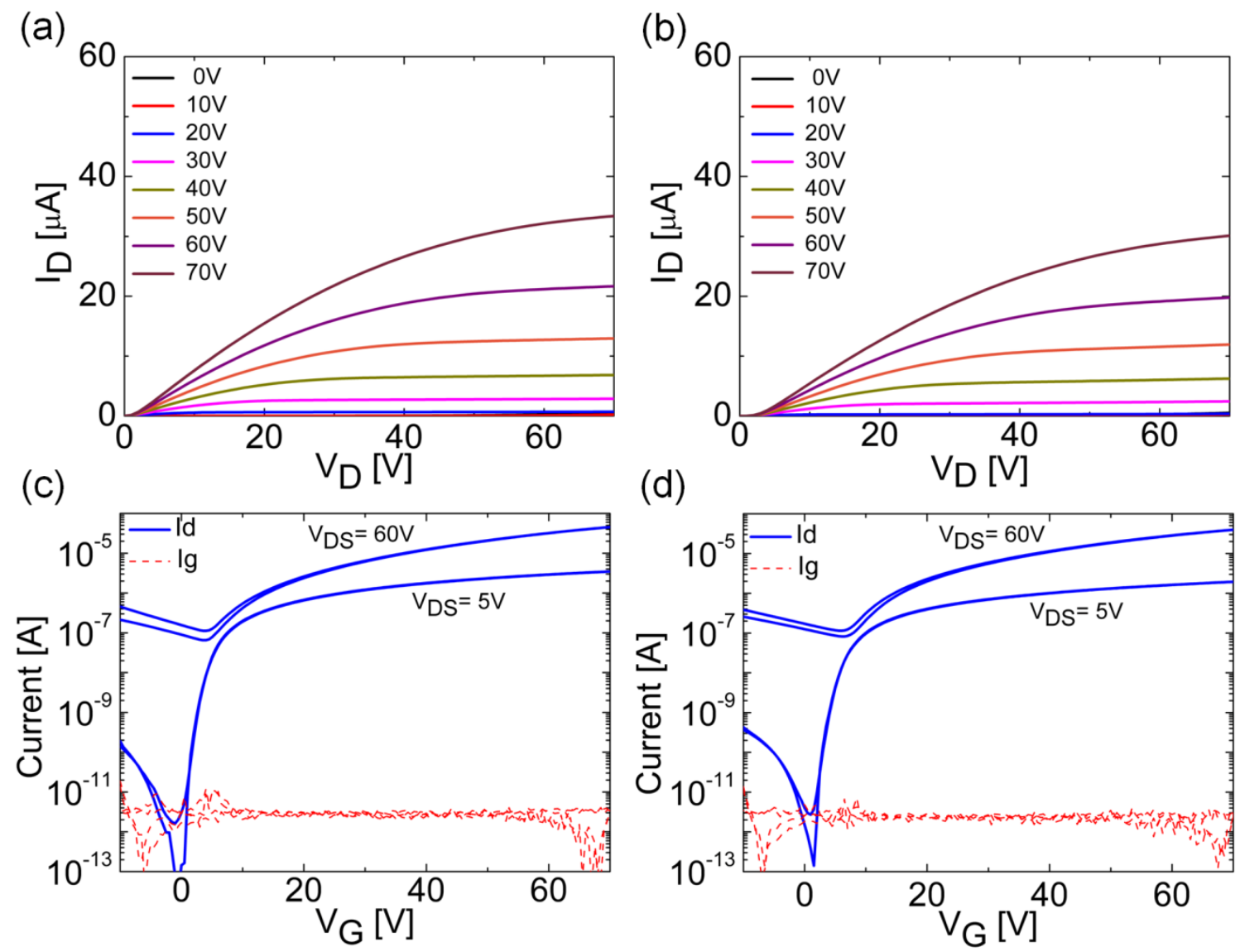

Figure S2. $n$-type printed P(NDI2OD-T2) FET characteristics. Output curves (a and b) and Transfer curves(c and d), based on printed PjetN electrodes (a and c) and ICP1050 (b and d). PjetN FET:W $=1210 \mu \mathrm{m} \mathrm{L}=55 \mu \mathrm{m}$; ICP1050 FET: $\mathrm{W}=1230 \mu \mathrm{m} \mathrm{L}=44 \mu \mathrm{m}$. 
(a)

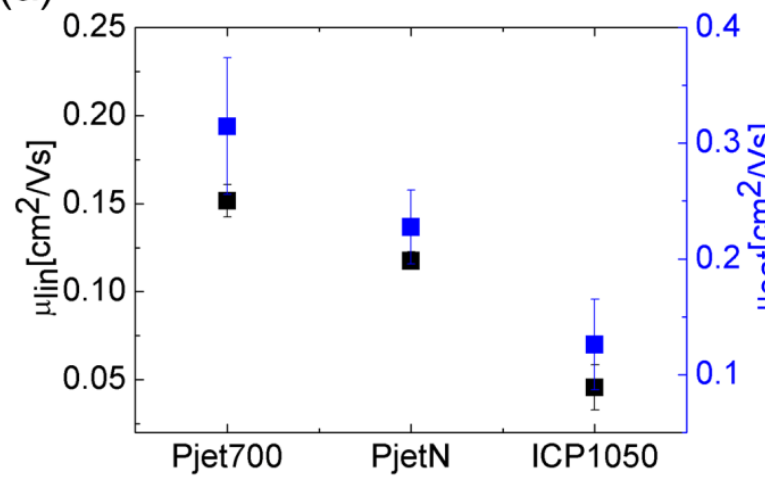

(b)

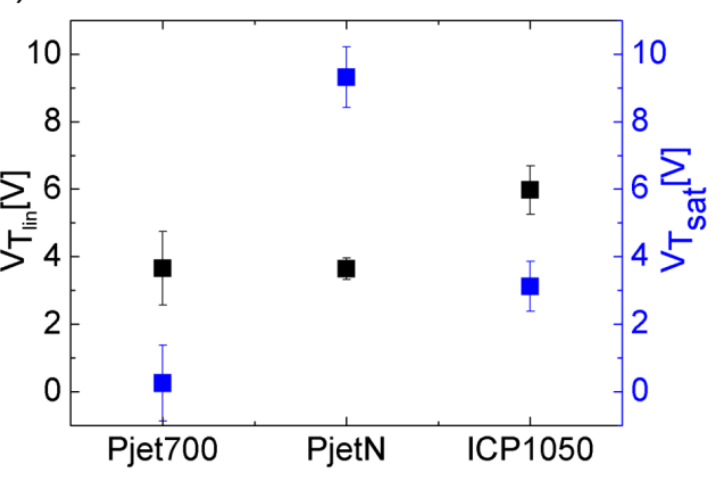

Figure S3. (a) Linear and saturation mobility and (b) linear and saturation threshold voltage of all three different PEDOT:PSS- and P(NDI2OD-T2)-based FETs. This study is based on eight devices for each different PEDOT:PSS formulation. Channel length was in the range of 50-60 micron. 
By considering the maximum current flowing in the devices throughout the measurements and the measured resistance of our contacts (Figure S1), an upper bound for the voltage drop along the source and drain contacts is calculated.

Table S1. Resistance along the contact, maximum drain current and maximum voltage drop along the single contact, of three different PEDOT:PSS formulations. The voltage drop is intentionally overestimated and it represents an upper bound; it is calculated as if the drain current was all collected at one edge of the contact and flowed through its entire length (corresponding to $W$ ). In reality, the voltage drop will be lower than this value.

\begin{tabular}{cccc}
\hline Formulation & $\begin{array}{c}\text { Resistance } \\
\text { along contact } \\
(\mathbf{k} \boldsymbol{\Omega})\end{array}$ & $\begin{array}{c}\text { Maximum } \\
\mathbf{I}_{\mathbf{d}}(\boldsymbol{\mu A})\end{array}$ & $\begin{array}{c}\text { Voltage drop } \\
\text { along single } \\
\text { contact }(\mathbf{V})\end{array}$ \\
\hline CleviosPjet700 & 15 & 53.11 & 0.4 \\
CleviosPjetN & 1020 & 32.03 & 16 \\
OrgaconICP1050 & 1975 & 28.46 & 27 \\
\hline
\end{tabular}

Contact angle measurement of all three different PEDOT:PSS on PEN substrate 
(c)

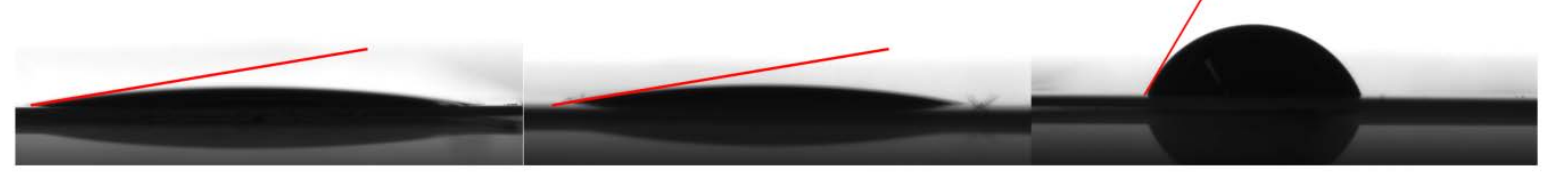

Figure S4. Contact angle of (a) CleviosPjet700, (b) CleviosPjetN and (c) OrgaconICP1050 on top of PEN substrate.

\section{Contact resistance measurement using differential method}

(a)

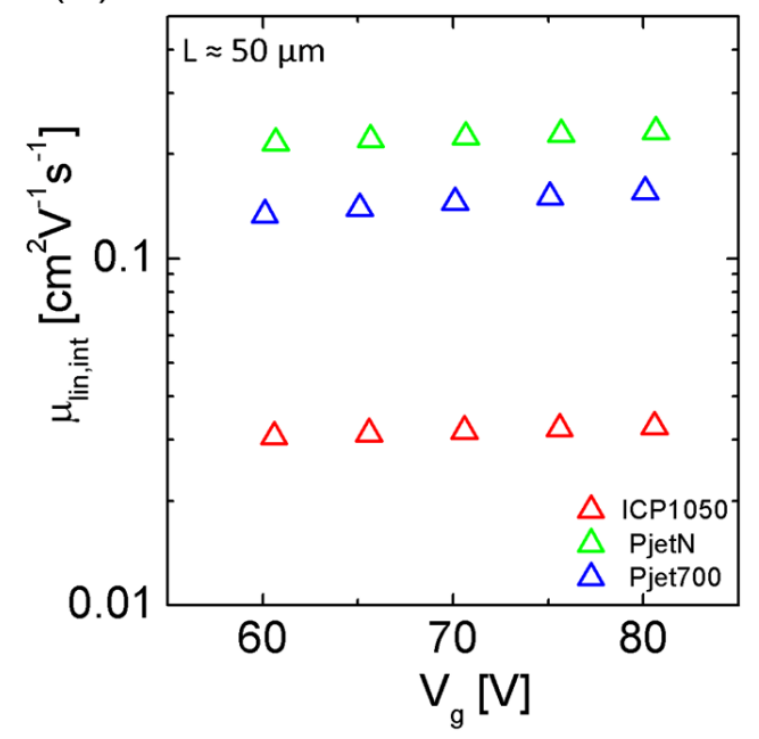

(b)

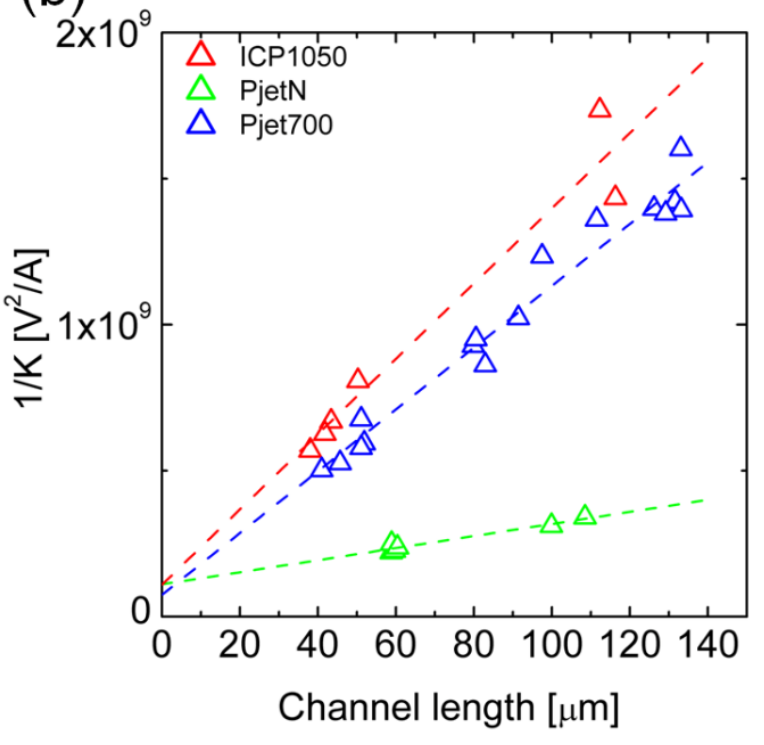

Figure S5. (a) Extraction of intrinsic linear mobility using differential method. (b) 1/K plot with channel length in differential method, where, $\frac{1}{K}=\frac{L}{\mu_{o} C_{o x} W}+\frac{\alpha}{W}, \mathrm{~W}, \mathrm{~L}$ and $\mathrm{C}_{\text {ox }}$ are channel width, channel length and dielectric capacitance per unit area. Slop and interaction at y-axis gives the values of $\mu_{0}$ and $\alpha$, details are given in [1].

$\underline{\text { AFM and Root Mean Square Roughness (R.M.S.) analysis of PEDOT:PSS and P(NDI2OD-T2) }}$ films 

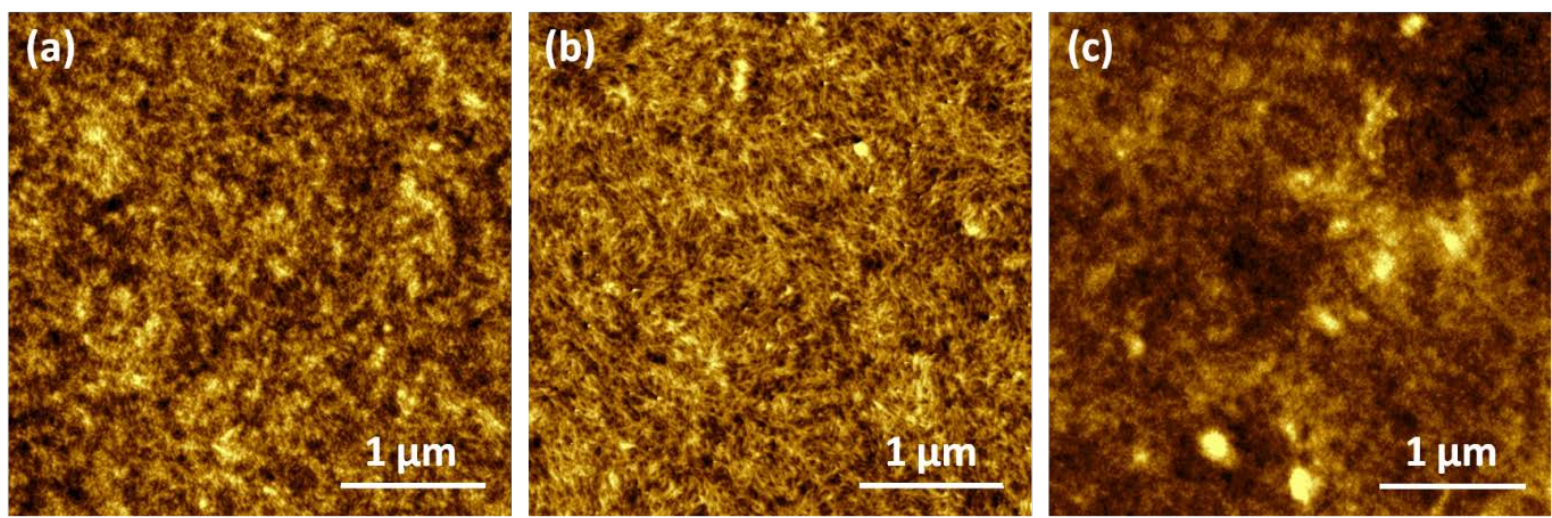

Figure S6. AFM image of (a) Clevios Pjet700 ( $\left.R_{\text {R.M.S. }}=2.41 \mathrm{~nm}\right)$, (b) Clevios PjetN (R.M.S. $=$ $3.09 \mathrm{~nm})$, and (c) Orgacon ICP1050 (R.M.M. $=3.20 \mathrm{~nm})$.
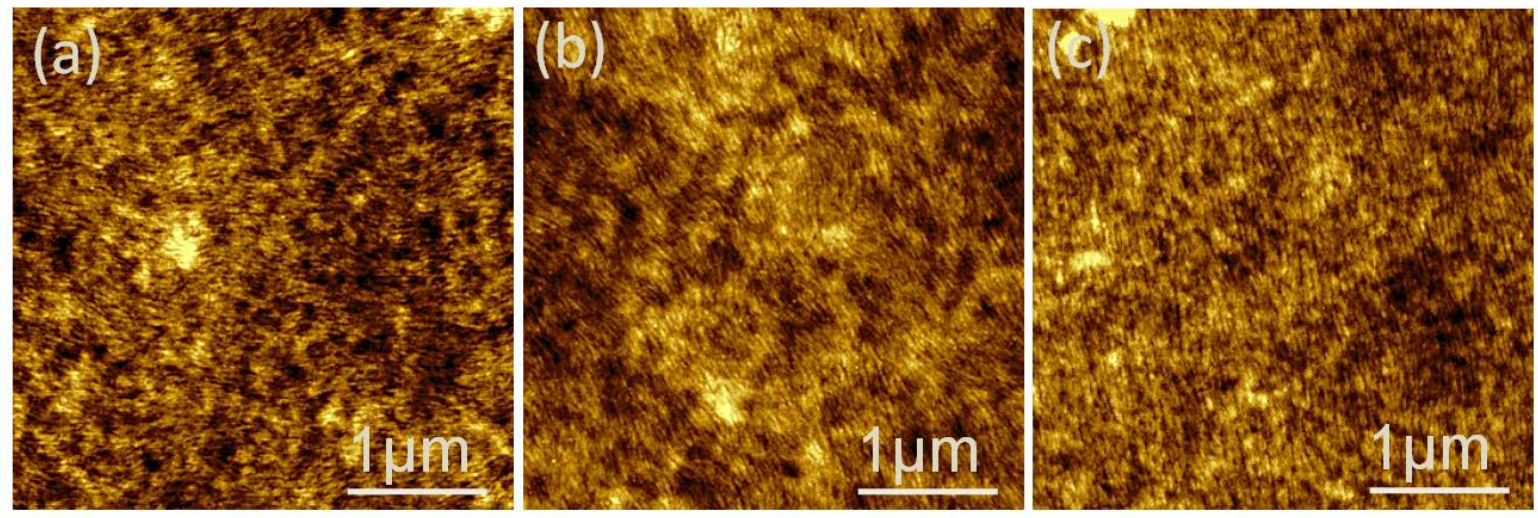

Figure S7. AFM image of P(NDI2OD-T2) on top of (a) Clevios Pjet700 ( $\left.R_{\text {R.M.S. }}=1.15 \mathrm{~nm}\right)$, (b) Clevios PjetN ( $\left.R_{R . M . S .}=1.45 \mathrm{~nm}\right)$, and (c) Orgacon ICP1050 $\left(R_{R . M . S .}=1.65 \mathrm{~nm}\right)$. 
Ultraviolet and x-ray photoelectron spectroscopy (UPS and XPS, respectively) were performed in a ultra-high vacuum system (base pressure $10^{-7} \mathrm{~Pa}$ ) equipped with a $150 \mathrm{~mm}$ hemispherical electron analyzer from SPECS GmbH. Electrons were excited with $\mathrm{Mg} \mathrm{Ka}$ radiation $(h v=1253.6 \mathrm{eV})$ for XPS, while UV radiation was provided by a helium discharge lamp (He I line, $h v=21.2 \mathrm{eV}$ ). The work function was estimated from the threshold energy for the emission of secondary electrons during He I excitation. In order to overcome the contact potential difference between the sample and the spectrometer, secondary electrons were accelerated by applying a negative potential $(-10 \mathrm{~V})$ to the sample.

Sample preparation: After the annealing step performed in an inert atmosphere, the PEDOT:PSS substrates were transferred in a nitrogen-filled enclosure to the photoelectron spectrometer and swiftly inserted into the vacuum system for the work function measurements. Due to the extremely small probing depth of UPS ( $1 \mathrm{~nm})$, a specific procedure was devised for evaluating the electronic structure of the polymer films from the interface to the bulk. Either P(NDI2OD-T2) or DPPT-TT were dissolved in mesitylene and spin coated on the annealed PEDOT:PSS substrates. The thickness of the polymer films was finely tuned by changing the polymer concentration in solution while keeping the other coating parameters fixed. Following this strategy, the sample coverage was varied from sub-monolayer to multilayer coverage [the thickness of one monolayer (ML) is $~ 2 \mathrm{~nm}$ ], up to a maximum thickness of $25-30 \mathrm{~nm}$. Atomic force microscopy (AFM) and XPS were employed to calibrate the coating parameters at low coverages, while a profilometer was used for thicker films. Spin coated films were baked and transferred to the spectrometer following the same procedure devised for the bare substrates.

Thickness calibration: Figure S8 shows a series of photoemission spectra acquired on PEDOT:PSS substrates coated with P(NDI2OD-T2) thin films of different thicknesses, produced 
by increasing the concentration of the polymer in solution from $0.5 \mathrm{mg} / \mathrm{ml}$ (bottom) to $3 \mathrm{mg} / \mathrm{ml}$ (top).

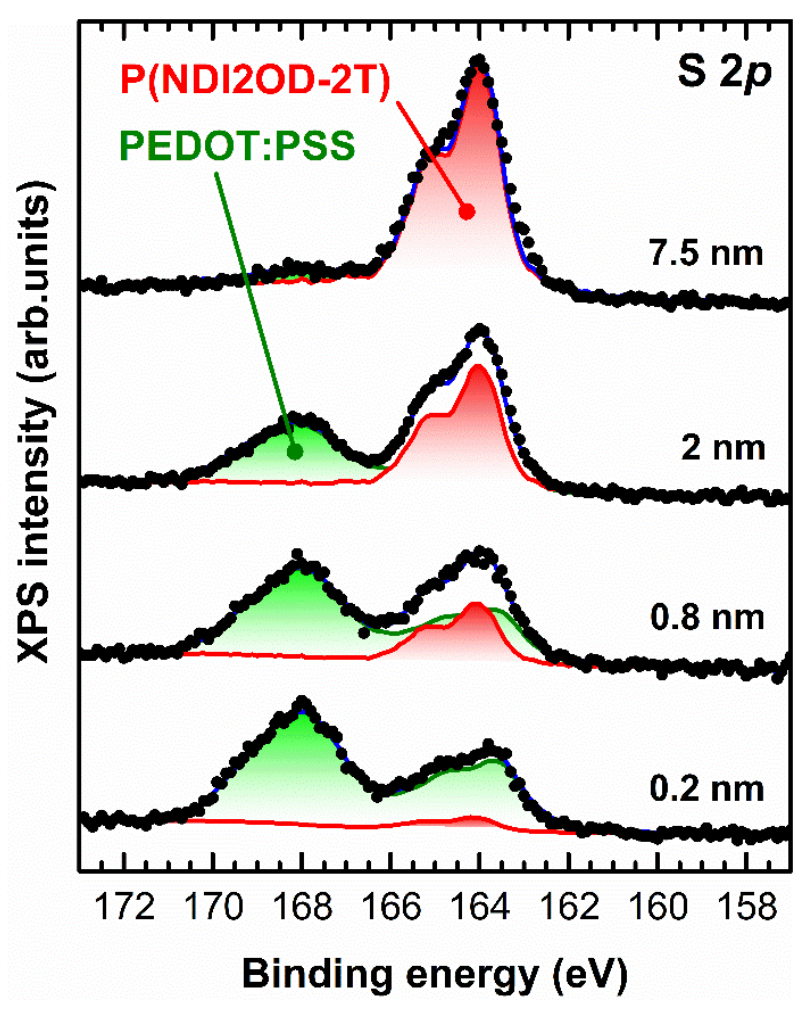

Figure S8. XPS scans of the S $2 p$ region, acquired from a series of samples with increasing $\mathrm{P}(\mathrm{NDI} 2 \mathrm{OD}-\mathrm{T2})$ coverage. The shaded red and green areas highlight the contribution of $\mathrm{P}(\mathrm{NDI} 2 \mathrm{OD}-\mathrm{T2})$ and PEDOT:PSS, respectively, to the $\mathrm{S} 2 p$ line shape. The thickness of the $\mathrm{P}(\mathrm{NDI} 2 \mathrm{OD}-\mathrm{T2}$ ) films is computed according to the model presented in the text.

The photoemission signal from the $\mathrm{S} 2 p$ core level is considered: $\mathrm{S}$ atoms in the thiophene units of P(NDI2OD-T2) give rise to a doublet peaking at a binding energy of about $164 \mathrm{eV}$ and highlighted in red in Figure S8. The photoemission spectrum of the PEDOT:PSS substrate is characterized by a more structured line shape (highlighted in green in Figure S8) resulting from the contribution of S atoms in PEDOT (at about $164 \mathrm{eV}$ ) and in PSS (at $168 \mathrm{eV}$ ) [2]. 
The ratio between the intensity of the P(NDI2OD-T2) and the PEDOT:PSS spectral components $\left(\frac{I_{\mathrm{P}(\mathrm{NDI} 2 \mathrm{OD}-\mathrm{T} 2)}}{I_{\text {PEDOT:PSS }}}\right)$ can be used to evaluate the P(NDI2OD-T2) film thickness $d$ according to the following equation [3]:

$d=\lambda \cdot \ln \left(\alpha \frac{I_{\mathrm{P}(\mathrm{NDI} 2 \mathrm{OD}-\mathrm{T} 2)}}{I_{\text {PEDOT:PSS }}}+1\right)$,

where $\lambda$ is the inelastic mean free path of the $\mathrm{S} 2 p$ photoelectrons through the $\mathrm{P}(\mathrm{NDI} 2 \mathrm{OD}-\mathrm{T} 2)$ film (about $3.6 \mathrm{~nm}$, as reported by Seah et al.[4]) and $\alpha$ is a constant term, determined from the S $2 p$ spectrum recorded on a sample with a defined P(NDI2OD-T2) film thickness (e.g. the one with a coverage of $1 \mathrm{ML}$, independently evaluated by AFM). The thickness reported for sub-ML coverages must be regarded as an average value accounting for the areas of the substrate covered by the P(NDI2OD-T2) layer and those areas left uncovered.

UPS characterization: Figure S9 reports the P(NDI2OD-T2)/PEDOT:PSS work function evolution as a function of the polymer layer thickness. Similar trends were observed for the HOMO level position and for the DPPT-TT/PEDOT:PSS system (data not reported here).

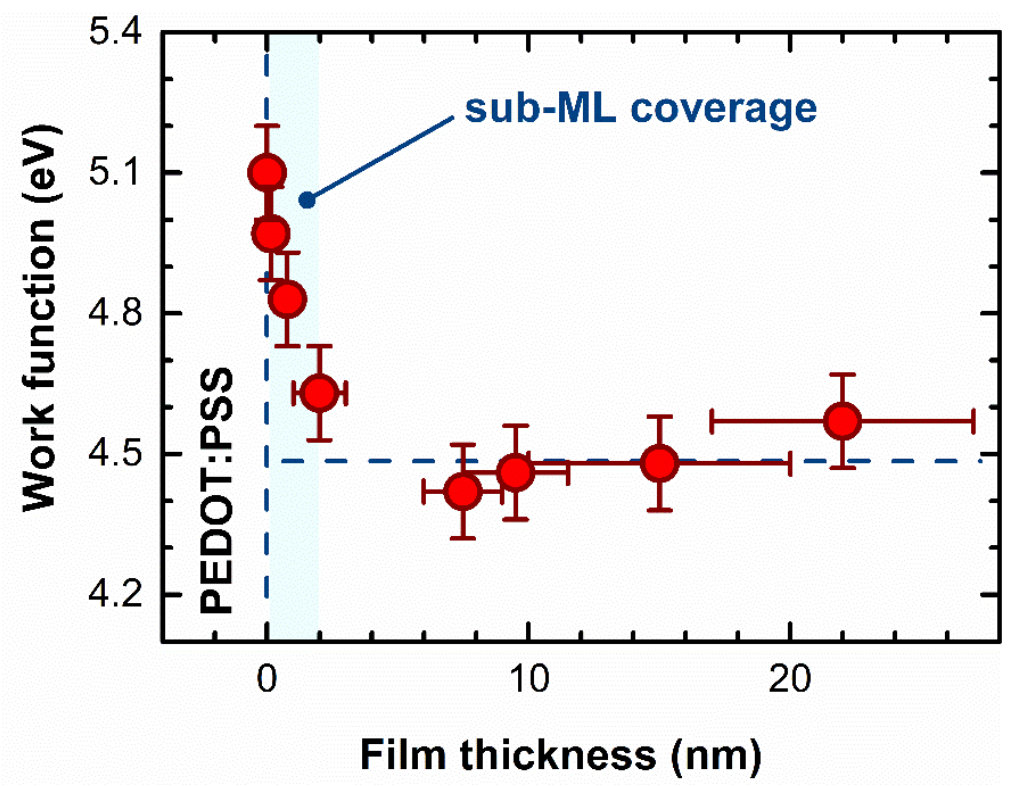

Figure S9. Work function vs. the P(NDI2OD-T2) film thickness on PEDOT:PSS. The experimental points enclosed in the shaded area refer to films thinner than $2 \mathrm{~nm}$ (1 ML sample). 
For each investigated thickness, a minimum of four individual work function and HOMO (highest occupied molecular orbital) level estimates were collected in different positions on the sample surface (about $10 \times 10 \mathrm{~mm}^{2}$ wide). The samples were irradiated for the time necessary to collect the photoemission spectra (about $20 \mathrm{~s}$ for each acquisition) by removing a shutter placed on the UV light path. We estimate an overall uncertainty of $0.1 \mathrm{eV}$ for the UPS results, accounting for the spectrometer resolution and for the sample-related variability. 
(a)

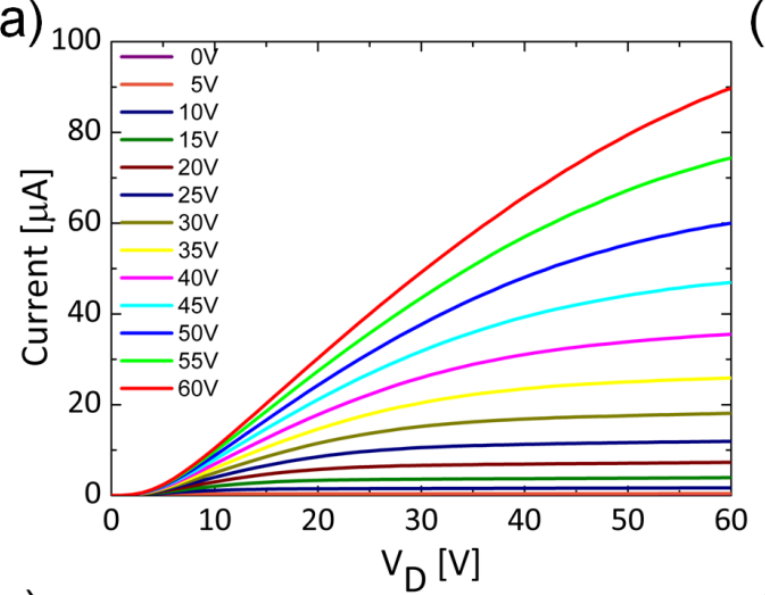

(c)

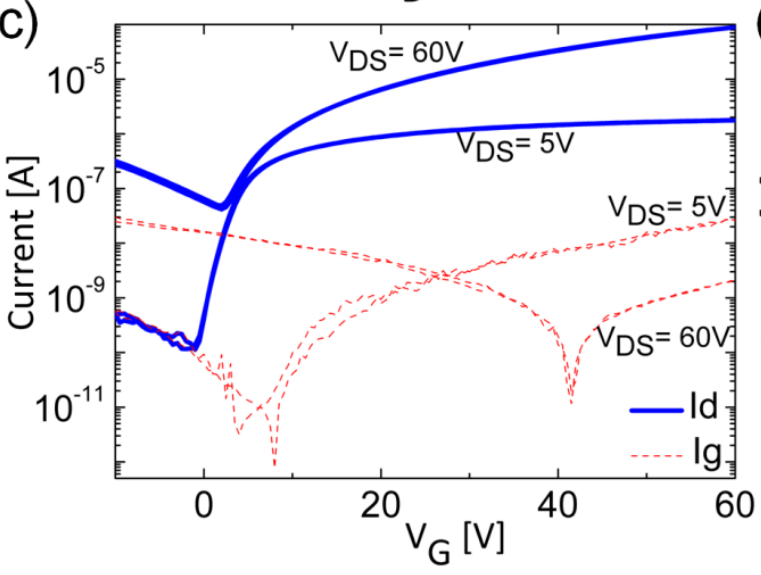

(b)

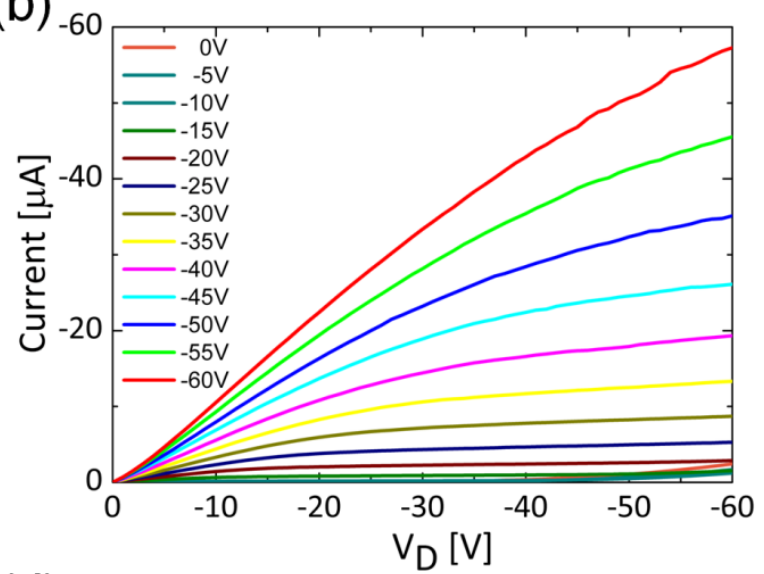

(d)

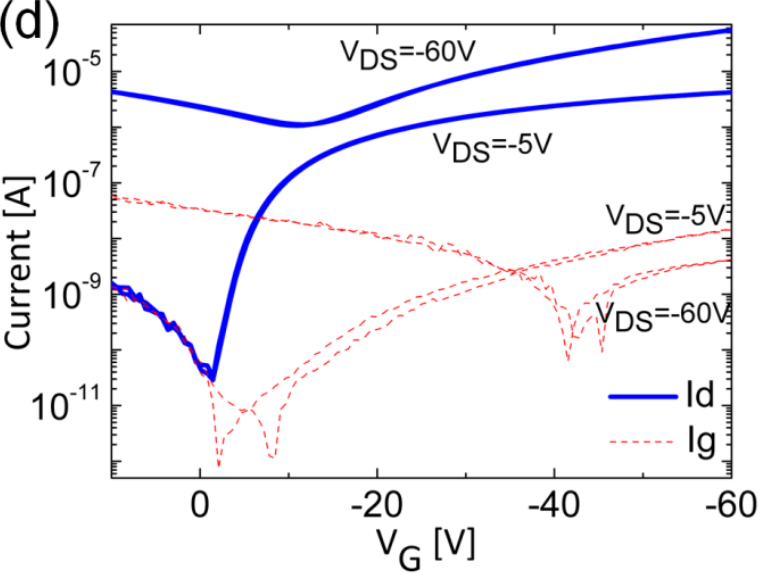

Figure S10. (a) \& (b) output characteristics and (c) \& (d) transfer characteristics of individual $\mathrm{P}(\mathrm{NDI} 2 \mathrm{OD}-\mathrm{T} 2)$ and DPPTTT OTFTs in the inverter. Aspect ratios of transistors are $\mathrm{W}_{\mathrm{p}} / \mathrm{L}_{\mathrm{p}}=$ $1200 \mu \mathrm{m} / 52 \mu \mathrm{m}$ and $\mathrm{W}_{\mathrm{n}} / \mathrm{L}_{\mathrm{n}}=1200 \mu \mathrm{m} / 45 \mu \mathrm{m}$. By measuring the transistors integrated in the inverter, higher gate leakages are found in each of the two devices because of the presence of additional leakage pathways through the complementary device. 


\section{Calculation of the gain of the inverter}

$\mathrm{g}_{\mathrm{m}}$ and $r_{\text {out }}$ are calculated using the following equations:

$g_{m p}=\frac{d I_{D p}}{d V_{g p}}$ and $g_{m n}=\frac{d I_{D n}}{d V_{g n}}$

And, $r_{o u t, p}=\frac{d V_{D p}}{d I_{D p}}$ and $r_{o u t, n}=\frac{d V_{D n}}{d I_{D n}}$

The logic inverting threshold was calculated from inverter VTC measurement, which was $30 \mathrm{~V}$ at $\mathrm{V}_{\mathrm{dd}}=60 \mathrm{~V}$ (Figure 6e).

In case of $\mathrm{P}(\mathrm{NDI} 2 \mathrm{OD}-\mathrm{T} 2)$ transistor in the inverter, the transconductance, $\mathrm{gmn}=4.43 \mathrm{x} 10$ ${ }^{4} \mathrm{~mA} / \mathrm{V}($ at $\mathrm{Vd}=30 \mathrm{~V})$ and the resistance ${ }_{\text {out }, n}=2.53 \times 10^{4} \mathrm{k} \Omega($ at $\mathrm{Vg}=30 \mathrm{~V})$

In case of DPPTTT transistor in the inverter, the transconductance, $\mathrm{g}_{\mathrm{mp}}=4.24 \times 10^{4}$ $\mathrm{mA} / \mathrm{V}($ at $\mathrm{Vd}=-30 \mathrm{~V})$ and the resistance, $r_{\text {out }, p}=5.33 \times 10^{4} \mathrm{k} \Omega($ at $\mathrm{Vg}=-30 \mathrm{~V})$.

Now, if we put the calculated values of $r_{\text {out }}$ and $\mathrm{g}_{\mathrm{m}}$ in the following equation:

$$
\text { Gain }=\frac{v_{\text {out }}}{v_{\text {in }}}=\left(g_{m p}+g_{m n}\right)\left(r_{\text {out }, p} \| r_{\text {out }, n}\right)=\left(g_{m p}+g_{m n}\right)\left(\frac{r_{\text {out }, p} r_{\text {out }, n}}{r_{\text {out }, p}+r_{\text {out }, n}}\right)=14.9 \frac{\mathrm{V}}{\mathrm{V}}
$$

This value is very close to the gain value obtained by extraction from inverter input-output curves, which is 14.1 (at $\left.V_{\mathrm{dd}}=60 \mathrm{~V}\right)$. 


\section{Frequency response of FET}

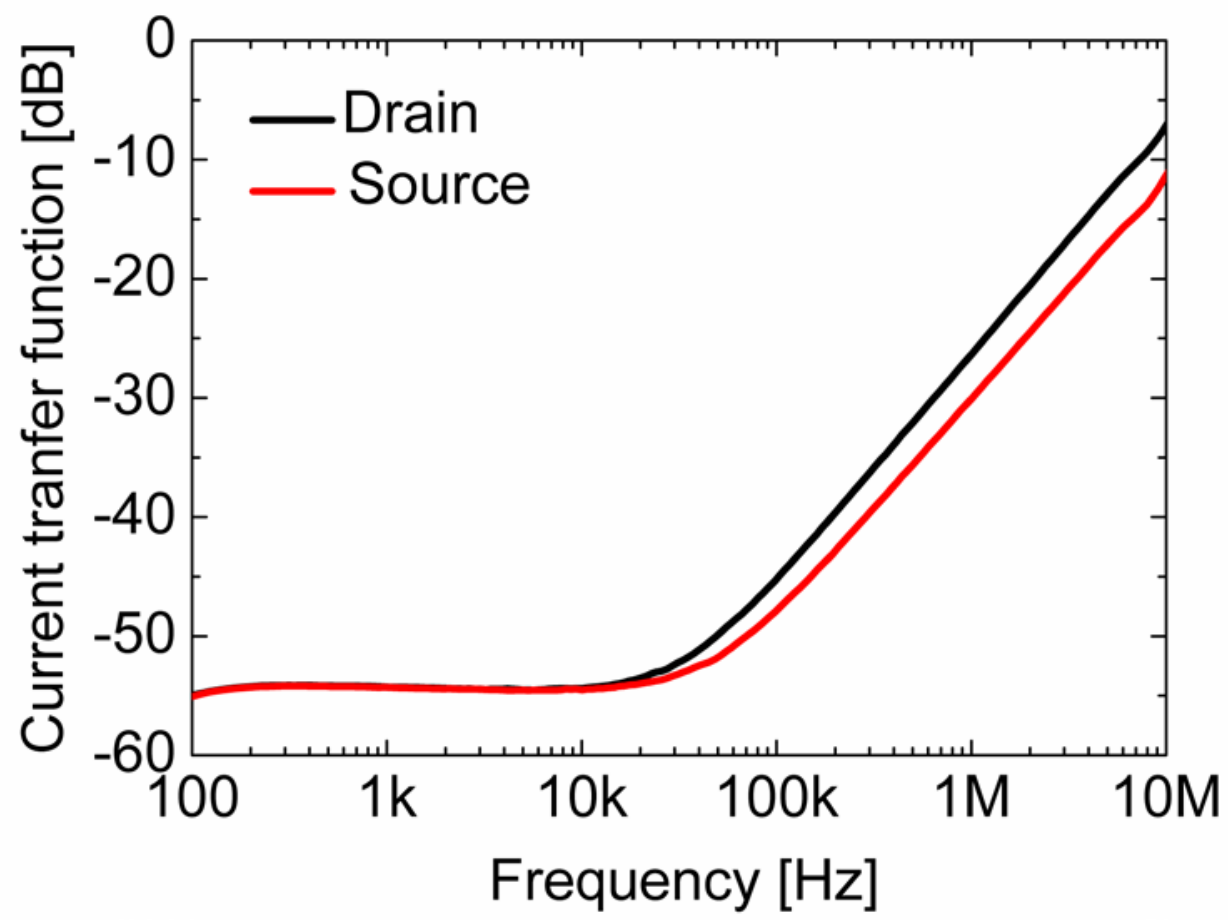

Figure S11. Admittance of P(NDI2OD-T2) based FET ( $\mathrm{L}=46 \mu \mathrm{m}, \mathrm{W}=1180 \mu \mathrm{m}$, dielectric thickness $=700 \mathrm{~nm}, \mathrm{~V}_{\mathrm{g}}=\mathrm{V}_{\mathrm{d}}=40 \mathrm{~V}$ ).

We have tested the frequency response of the printed transistors by measuring their transition frequency $\left(f_{\mathrm{T}}\right)$, defined as [5]

$$
f_{T}=\frac{g_{m}}{2 \pi\left(C_{g s}+C_{g d}\right)}
$$

By using a custom measurement setup, based on Agilent Technologies E5061B Network Analyzer and schematized in Fig. S12, the admittance of the device is measured separately on the source and drain sides (Figure S11). During the measurement, an appropriate DC bias is applied to each terminal. The transconductance and parasitic capacitances between the gate and each contact are extracted using these measurements. 


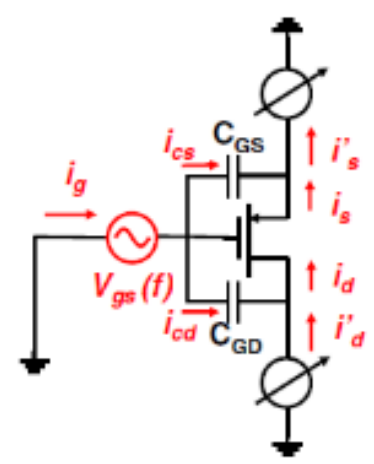

Figure S12. Schematic of the setup for the transition frequency measurement [5].

The admittance of a transistor with a 46 $\mu \mathrm{m}$ long channel, measured in transdiode configuration, is shown in Figure S11. At low frequencies, up to $\approx 20 \mathrm{kHz}$, the device behaves as a constant current generator, and its transconductance $\left(g_{\mathrm{m}}\right)$ can be extracted through:

$$
g_{m}=\frac{10^{\frac{Z}{20}}}{G}=0.961372 \mu \mathrm{A} / \mathrm{V}
$$

where $\mathrm{Z}$ is the measured admittance in $\mathrm{dB}$, and $\mathrm{G}=2 * 10^{3}$ is the gain of the transimpedance of the measurement setup.

In the high frequency region (over $20 \mathrm{kHz}$ ) the admittance is dominated by the capacitive coupling between the gate and source (drain) contacts, allowing the value of these two capacitances to be independently determined: $\mathrm{C}_{\mathrm{gs}}=2.347049 \mathrm{pF}, \mathrm{C}_{\mathrm{gd}}=3.814062 \mathrm{pF}$.

The transition frequency of the device was calculated using the following formula:

$$
f_{T}=\frac{g_{m}}{2 \pi\left(C_{g s}+C_{g d}\right)}=24.85 \mathrm{kHz}
$$


Frequency response of all printed all polymer inverter.

(a)
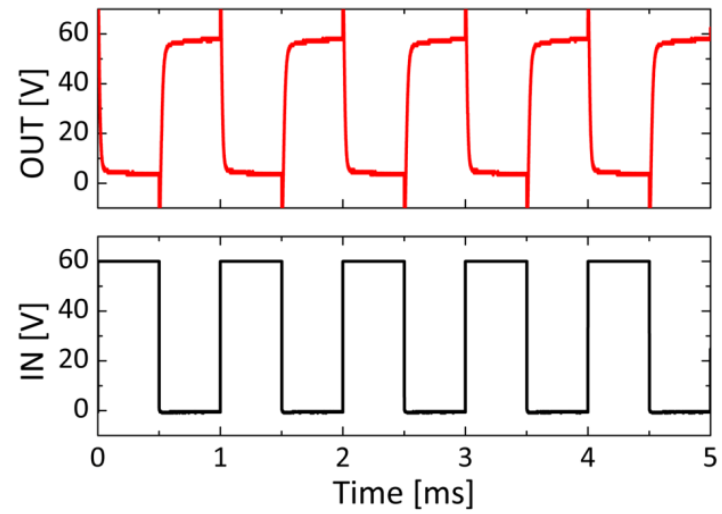

(c)
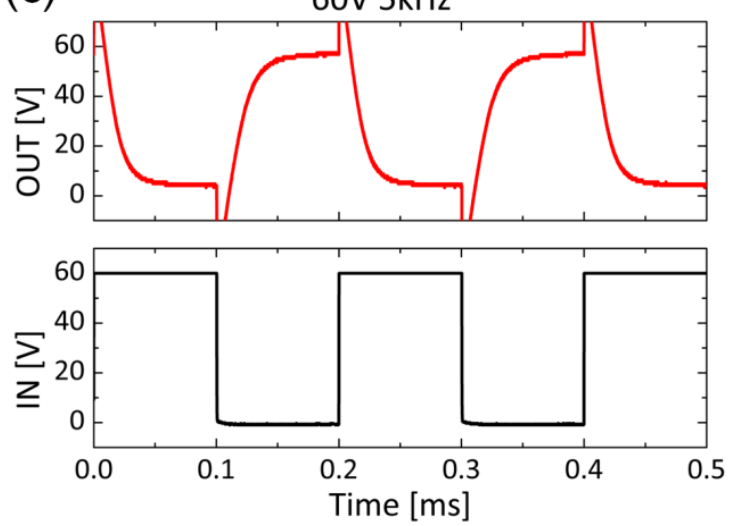

(b)
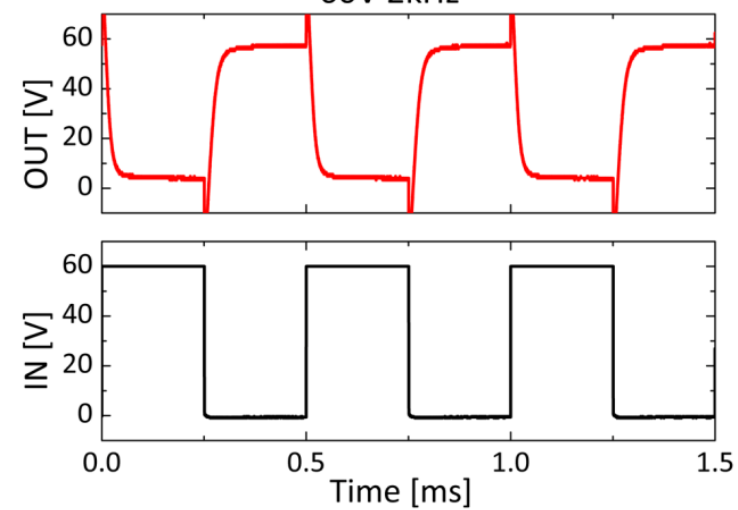

(d)
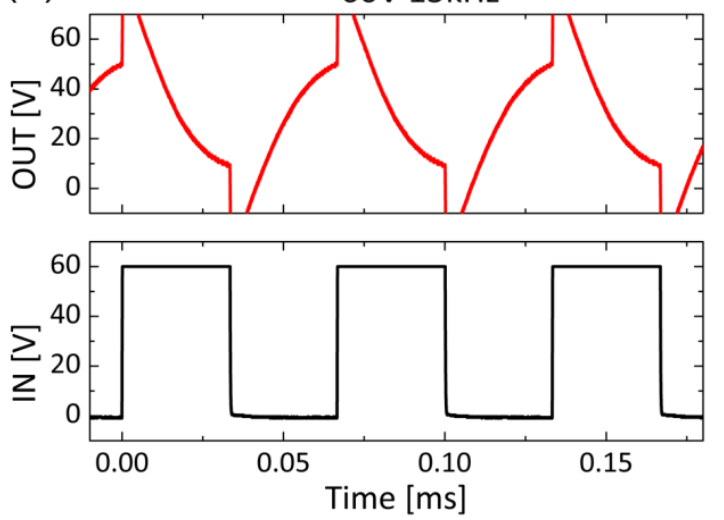

Figure S13. Dynamic response of a complementary inverter based on P(NDI2OD-T2) and DPPT-TT to (a) $1 \mathrm{kHz}$, (b) $2 \mathrm{kHz}$, (c) $5 \mathrm{kHz}$ and (d) $15 \mathrm{kHz}$ square wave. Aspect ratios of transistors are $\mathrm{W}_{\mathrm{p}} / \mathrm{L}_{\mathrm{p}}=1200 \mu \mathrm{m} / 52 \mu \mathrm{m}$ and $\mathrm{W}_{\mathrm{n}} / \mathrm{L}_{\mathrm{n}}=1200 \mu \mathrm{m} / 45 \mu \mathrm{m}$. 


\section{All printed all polymer 3-stage ring oscillators}

(a)

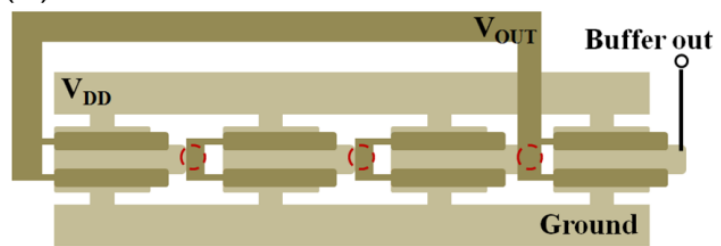

(c) 40

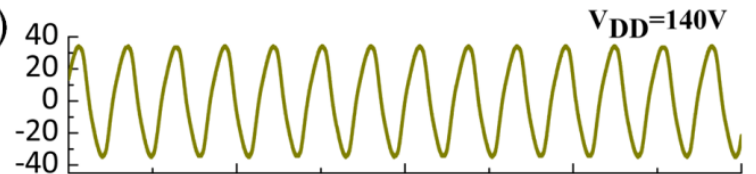

(b)
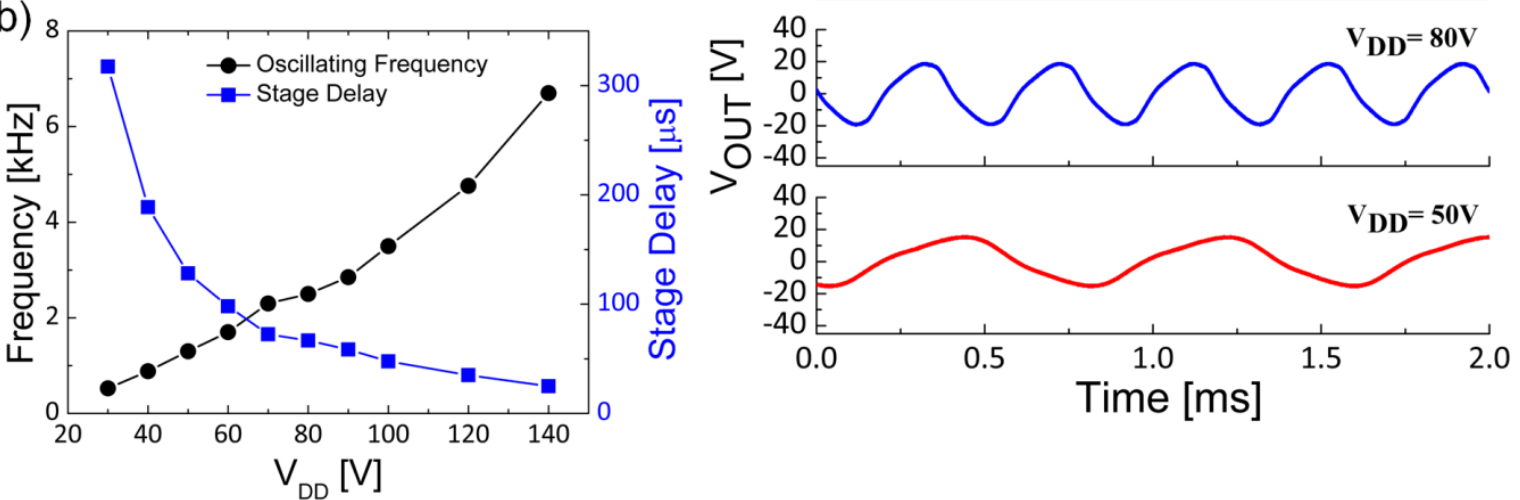

Figure S14. (a) Schematic of 3-stage ring oscillator, (b) oscillating frequency and stage delay vs supply voltage and (c) output signal of a three stage ring oscillator at supply voltage of $50 \mathrm{~V}, 80 \mathrm{~V}$ and $140 \mathrm{~V}$. 


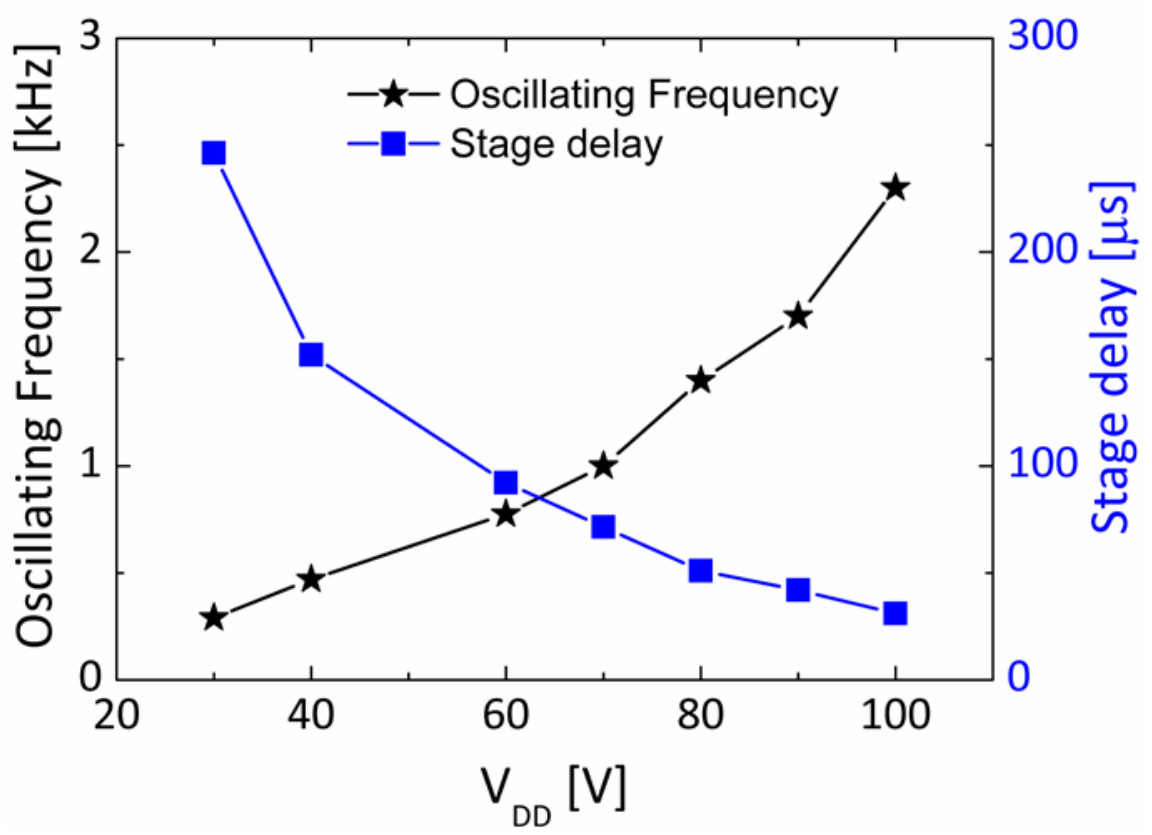

Figure S15. Oscillating frequency and stage delay of a seven stage ring oscillator as a function of supply voltage. 


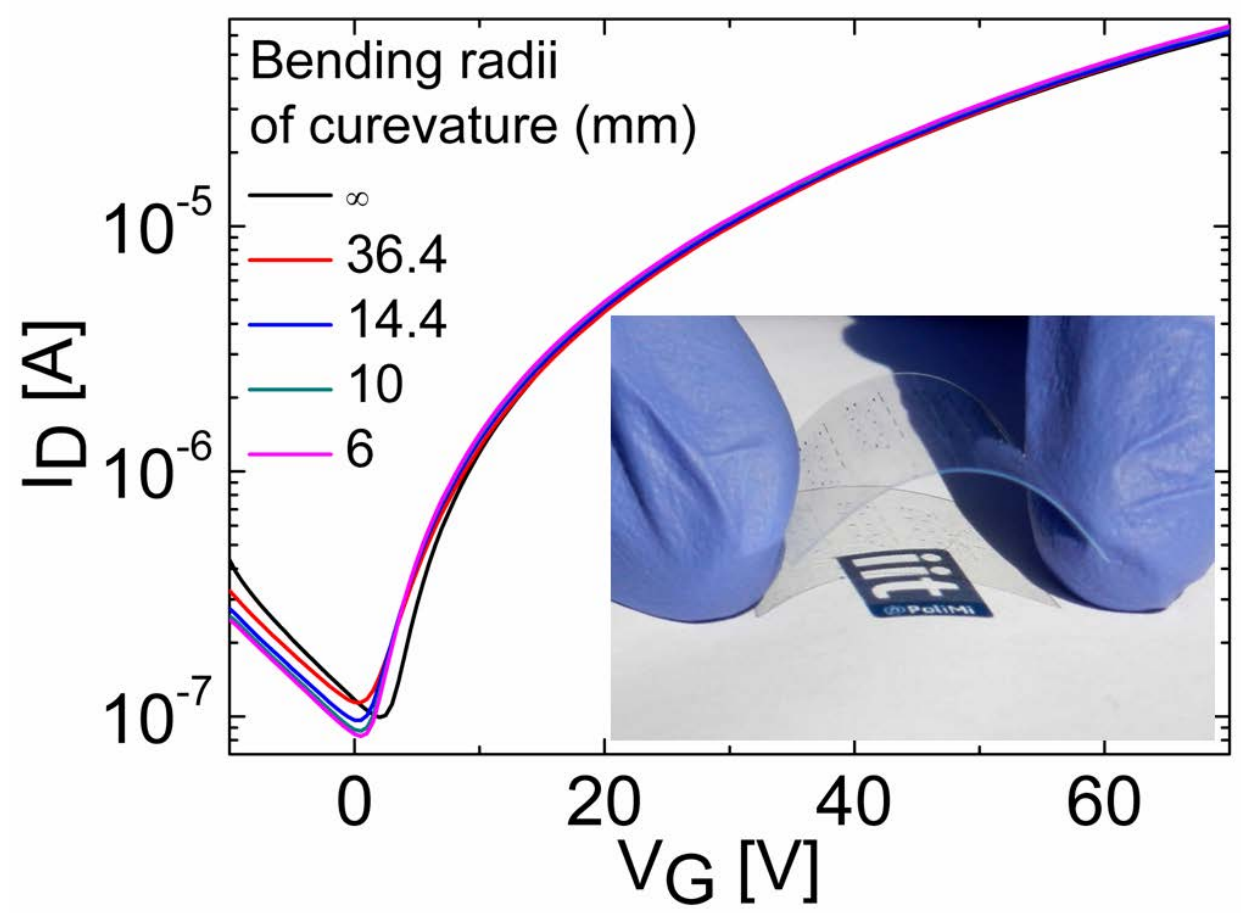

Figure S16. Transfer curve of Pjet700-P(NDI2OD-T2) OTFT vs different bending radii of curvature.

[1] D. Natali, L. Fumagalli, M. Sampietro, J. Appl. Phys. 101 (2007) 014501.

[2] G. Greczynski, Th. Kugler, M. Keil, W. Osikowicz, M. Fahlman, W.R. Salaneck, J. Electron. Spectrosc. Relat. Phenom. 121 (2001) 1-17.

[3] S. Hofmann, Auger and X-ray Photoelectron Spectroscopy, $2^{\text {nd }}$ Edition, Practical Surface Analysis (Eds: D. Briggs, M.P. Seah), Wiley, New York 1 (1996) 143.

[4] M. P. Seah, W. A. Dench, Surf. Interface Anal.1 (1979) 2-11.

[5] M. Caironi, Y-Y. Noh, H. Sirringhaus, Semicond. Sci. Technol., 26 (2011) 034006. 